\title{
THE GENERAL LIMIT THEOREM IN THE THEORY OF PROBABILITY ${ }^{1}$
}

\section{WOLFGANG STERNBERG}

Let $x_{1}, x_{2}, \cdots, x_{n}$ be independent chance variables with any distribution functions $V_{1}, V_{2}, \cdots, V_{n}$.

We put ${ }^{2}$ for all $i=1,2, \cdots, n$

$$
M\left[x_{i}\right]=\int \xi d V_{i}(\xi)=a_{i} \quad \text { (mean value) }
$$

St. D. $\left(x_{i}\right)=\int\left(\xi-a_{i}\right)^{2} d V_{i}(\xi)=b_{i} \quad$ (standard deviation),

$\int\left|\xi-a_{i}\right|{ }^{3} d V_{i}(\xi)=c_{i} \quad$ (absolute moment of the 3rd order).

(The term "standard deviation" is used by some authors for $b_{i}^{1 / 2}$ instead of $b_{i}$.)

Denoting mean value and standard deviation of the sum $x_{1}+\cdots+x_{i}(i=2, \cdots, n)$ by $A_{i}$ and $B_{i}$ respectively, it is known that

$$
A_{i}=a_{1}+\cdots+a_{i}, \quad B_{i}=b_{1}+\cdots+b_{i} .
$$

For the distribution function $W_{i}$ of the sum $x_{1}+\cdots+x_{i}$ the recursion formula

$$
W_{i}(x)=\int W_{i-1}(x-\xi) d V_{i}(\xi), \quad i>1,
$$

holds true.

Let $a_{i}$ and $B_{n}$ be as follows:

$$
\begin{aligned}
a_{i} & =0, \\
B_{n} & =1 .
\end{aligned}
$$

These conditions are only preliminarily stated in order to abbreviate the explanation. From (2) we have that $A_{i}=0$, in particular, $A_{n}=0$. (The case $b_{i}=0$ may be excluded, since then $x_{i}$ assumes the value 0 only with the probability 1 and thus does not appear in the sum $x_{1}+\cdots+x_{n}$.)

1 Presented to the Society, October 28, 1939.

2 The following integrals are Stieltjes integrals. The lower and upper limits always are $-\infty$ and $+\infty$ respectively. 
We have to prove that the function $W_{n}(x)$ lies uniformly in $x$ near the Gaussian function having also the mean value 0 and the standard deviation 1 , that is,

$$
\phi\left(x / 2^{1 / 2}\right)=\frac{1}{\pi^{1 / 2}} \int_{-\infty}^{x / 2^{1 / 2}} e^{-t^{2}} d t
$$

if very general suppositions with respect to the $V$ are made. We suppose that the quotients $c_{i} / b_{i}$ are "very small" for all $i$ and state the general limit theorem in the following form:

To every positive $\epsilon$ there is a positive $\delta$, so that uniformly in $x$

$$
\left|W_{n}(x)-\phi\left(x / 2^{1 / 2}\right)\right|<\epsilon
$$

\section{holds true if the conditions}

$$
c_{i} / b_{i}<\delta, \quad i=1,2, \cdots, n,
$$

are satisfied.

Our proof is suggested by that of A. Khintchine (see below). We remark first of all that

$$
b_{i}<\delta, \quad i=1,2, \cdots, n,
$$

follows from (4) according to the well known inequality $b_{i}^{3}<c_{i}^{2}$. (Of course we can suppose $\delta<1$.) Since $B_{n}=1$ but the $b_{i}$ are small, it is obvious that $n$ must be a large number.

Now we note that the function of two variables

$$
\phi\left(x /(2 y)^{1 / 2}\right)=\frac{1}{\pi^{1 / 2}} \int_{-\infty}^{x /(2 y)^{1 / 2}} e^{-t^{2}} d t=U(x, y)
$$

that is, the Gaussian function with the variable standard deviation $y$ represents a solution of the differential equation of the conduction of heat

$$
\frac{\partial \phi}{\partial y}=\frac{1}{2} \frac{\partial^{2} \phi}{\partial x^{2}}
$$

Here and in what follows it is always supposed that $y \geqq \eta, \eta$ being a positive small but constant magnitude. The equation (1) suggests that we compare the two expressions $U\left(x, B_{i}\right)$ and

$$
\int U\left(x-\xi, B_{i-1}\right) d V_{i}(\xi)
$$

(Later the variable $y$ will be put equal to $B_{i-1}$.) According to Taylor's 
formula we have

$$
\begin{aligned}
U\left(x, y+b_{i}\right) & =U(x, y)+b_{i} \frac{\partial U(x, y)}{\partial y}+R_{1}, \\
R_{1} & =\frac{b_{i}^{2}}{2} \frac{\partial^{2} U\left(x, y+\theta b_{i}\right)}{\partial y^{2}}, \quad 0<\theta<1 .
\end{aligned}
$$

Let $M(\eta)$ be an upper limit of the absolute values of the derivatives of the $2 \mathrm{~d}$ and $3 \mathrm{~d}$ order of $U(x, y)$ in the half-plane $y \geqq \eta$; then

$$
\left|R_{1}\right|<b_{i}^{2} M(\eta) / 2 \text {. }
$$

On the other hand,

$$
\begin{aligned}
U(x-\xi, y)= & U(x, y)-\xi \frac{\partial U(x, y)}{\partial x}+\frac{\xi^{2}}{2} \frac{\partial^{2} U(x, y)}{\partial x^{2}} & \\
& -\frac{\xi^{3}}{6} \frac{\partial^{3} U\left(x-\theta_{1} \xi, y\right)}{\partial x^{3}}, & 0<\theta_{1}<1 .
\end{aligned}
$$

Therefore, in view of (2) and (6)

$$
\int U(x-\xi, y) d V_{i}(\xi)=U(x, y)+b_{i} \frac{\partial U(x, y)}{\partial y}+R_{2},
$$

where

$$
R_{2}=-\frac{1}{6} \int \xi^{3} \frac{\partial^{3} U\left(x-\theta_{1} \xi, y\right)}{\partial x^{3}} d V_{i}(\xi)
$$

thus

$$
\left|R_{2}\right|<c_{i} M(\eta) / 6
$$

It follows that

$$
U\left(x, y+b_{i}\right)=\int U(x-\xi, y) d V_{i}(\xi)+R
$$

in which $R=R_{1}-R_{2}$; accordingly

(8) $|R| \leqq\left|R_{1}\right|+\left|R_{2}\right|<M(\eta)\left\{b_{i}^{2} / 2+c_{i} / 6\right\}<[M(\eta) / 2]\left\{b_{i}^{2}+c_{i}\right\}$.

Now note that all $b_{i}<\eta$ because of (5), since of course $\delta<\eta$ may be supposed. On the contrary there are numbers $B_{i}>\eta$, as for instance $B_{n}=1>\eta$. There must be consequently a number $B_{r}$ satisfying $\eta<B_{r}<2 \eta$. Now we put $y=B_{i-1}$ in (7); and we suppose $i \geqq r+1$ in order that $y \geqq B_{r}>\eta$. Besides we write with regard to what follows 
$x+2 \alpha$ instead of $x, \alpha$ representing a positive parameter. Then it follows from (7) that

$$
U\left(x+2 \alpha, B_{i}\right)=\int U\left(x+2 \alpha-\xi, B_{i-1}\right) d V_{i}(\xi)+R
$$

in which $R$ satisfies (8). Putting the difference

$$
\begin{aligned}
W_{i}(x)-U\left(x+2 \alpha, B_{i}\right)=W_{i}(x)-\phi\left(\frac{x+2 \alpha}{\left(2 B_{i}\right)^{1 / 2}}\right) & =D_{i}(x), \\
i & =1,2, \cdots, n,
\end{aligned}
$$

and subtracting (9) from (1), we get the fundamental formula, valid for all $x$,

$$
D_{i}(x)=\int D_{i-1}(x-\xi) d V_{i}(\xi)-R
$$

together with (8), where $i=r+1, r+2, \cdots, n$.

In order to get further estimates we need the elementary inequalities of Tschebyscheff, which are valid for any distribution function $F$ with vanishing mean value and standard deviation not greater than $B$ :

$$
F(\alpha) \geqq 1-B / \alpha^{2}, \quad F(-\alpha) \leqq B / \alpha^{2}, \quad \alpha>0 .
$$

From these inequalities it easily follows for two distribution functions $F_{1}, F_{2}$ with vanishing mean values, the standard deviations of which are both not greater than $B$, and for all $x$, that

$$
F_{1}(x)-F_{2}(x+2 \alpha) \leqq B / \alpha^{2}, \quad \alpha>0 .
$$

For, if $x \leqq-\alpha$, one has $F_{1}(x) \leqq F_{1}(-\alpha) \leqq B / \alpha^{2}$; thus (12) follows because $F_{2} \geqq 0$. If $x>-\alpha$, one concludes $F_{2}(x+2 \alpha) \geqq F_{2}(\alpha) \geqq 1-B / \alpha^{2}$; therefore

$$
F_{1}(x)-F_{2}(x+2 \alpha) \leqq 1-F_{2}(x+2 \alpha) \leqq 1-\left(1-B / \alpha^{2}\right),
$$

that is, (12). On the foundation of (11) and (12) the proof may be carried out. Putting $i=r$ one has, according to the definition of $D_{i}(x)$ and because both $W_{r}(x)$ and $U\left(x, B_{r}\right)$ have the mean value 0 and the standard deviation $B_{r}<2 \eta$, on account of (12),

$$
W_{r}(x)-U\left(x+2 \alpha, B_{r}\right)=D_{r}(x)<2 \eta / \alpha^{2} \quad \text { for all } x, \quad \alpha>0 ;
$$

whence, denoting by $g_{i}$ the upper limit of $D_{i}(x)$, we conclude from (13) $g_{r}<2 \eta / \alpha^{2}$ and from (11), for any $i \geqq r+1, D_{i}(x) \leqq g_{i-1}+|R|$; thus

$$
g_{i} \leqq g_{i-1}+[M(\eta) / 2]\left\{b_{i}^{2}+c_{i}\right\}
$$


and through induction from $i$ to $i+1$

$$
g_{n} \leqq g_{r}+\frac{M(\eta)}{2} \sum_{i=r+1}^{n}\left\{b_{i}^{2}+c_{i}\right\}<\frac{2 \eta}{\alpha^{2}}+\frac{M(\eta)}{2} \sum_{i=1}^{n}\left\{b_{i}^{2}+c_{i}\right\} .
$$

On the other hand one has

$$
b_{i}<\delta, \quad c_{i} / b_{i}<\delta, \quad i=1,2, \cdots,
$$

because of (5) and (4). Therefore

$$
\sum_{i=1}^{n} b_{i}^{2}<\delta \sum b_{i}=\delta B_{n}=\delta, \quad \sum_{i=1}^{n} c_{i}<\delta \sum b_{i}=\delta .
$$

It follows that $g_{n}<2 \eta / \alpha^{2}+\delta M(\eta)$; thus

$$
D_{n}(x)=W_{n}(x)-\phi\left(\frac{x+2 \alpha}{2^{1 / 2}}\right)<2 \eta / \alpha^{2}+\delta M(\eta) .
$$

Therefore

$$
W_{n}(x)<\phi\left(x / 2^{1 / 2}\right)+2 \alpha /(2 \pi)^{1 / 2}+2 \eta / \alpha^{2}+\delta M(\eta) .
$$

The positive number $\epsilon$ being given, one determines first $\alpha$ through $2 \alpha /(2 \pi)^{1 / 2}=\epsilon / 3$, then $\eta$ through $2 \eta / \alpha^{2}=\epsilon / 3$, with which $M(\eta)$ is determined, and finally $\delta$ through $\delta M(\eta) \leqq \epsilon / 3$. It follows that

$$
W_{n}(x)<\phi\left(x / 2^{1 / 2}\right)+\epsilon,
$$

uniformly in $x$. Using

$$
\phi\left(\frac{x-2 \alpha}{\left(2 B_{i}\right)^{1 / 2}}\right)-W_{i}(x)=\Delta_{i}(x)
$$

one finds in the same way

$$
\Delta_{n}(x)<2 \eta / \alpha^{2}+\delta M(\eta)
$$

and

$$
W_{n}(x)>\phi\left(x / 2^{1 / 2}\right)-\epsilon .
$$

The proof is thus complete.

One should compare this with the proof given by A. Khintchine (Asymptotische Gesetze der Wahrscheinlichkeitsrechnung, Ergebnisse der Mathematik und ihrer Grenzgebiete, vol. 2, no. 4, Berlin, 1933), which is based on works of I. Petrowsky and A. Kolmogoroff. We have chosen other assumptions in order to make the exposition as simple as possible, while Khintchine strives for wide generality so that nothing is supposed by him as to moments of the third order. 
A so-called upper- and lower-function, the introduction of which is denoted by Khintchine as the fundamental idea of the PetrowskyKolmogoroffian proof, is not used by us.

We now generalize the investigation and omit the special conditions (2) and (3).

We use the transformation

$$
\frac{x_{i}-a_{i}}{B_{n}^{1 / 2}}=u_{i}, \quad i=1, \cdots, n .
$$

If mean value, standard deviation, and absolute moment of the third order of $u_{i}$ are denoted by $\bar{a}_{i}, \bar{b}_{i}$, and $\bar{c}_{i}$ respectively, it becomes

$$
\bar{a}_{i}=0, \quad \bar{b}_{i}=b_{i} / B_{n}, \quad \bar{c}_{i}=c_{i} /\left(B_{n}\right)^{3 / 2}
$$

and accordingly

and

$$
\frac{\bar{c}_{i}}{\bar{b}_{i}}=\frac{c_{i}}{b_{i}\left(B_{n}\right)^{1 / 2}}
$$

$$
\bar{B}_{n}=\text { St. D. }\left(u_{1}+\cdots+u_{n}\right)=\bar{b}_{1}+\cdots+\bar{b}_{n}=1 \text {. }
$$

The assumptions (4) must be replaced by

$$
\frac{c_{i}}{b_{i} B_{n}^{1 / 2}}<\delta, \quad i=1, \cdots, n .
$$

Denoting by $\bar{W}_{n}$ the distribution function of the sum $u_{1}+\cdots+u_{n}$, we thus have according to (16), (17) uniformly in $u$

$$
\bar{W}_{n}(u)=\phi\left(u / 2^{1 / 2}\right)+\sigma, \quad|\sigma|<\epsilon .
$$

But it easily follows in view of

$$
u_{1}+\cdots+u_{n}=\left(x_{1}+\cdots+x_{n}-A_{n}\right) / B_{n}^{1 / 2}
$$

that the distribution function $W_{n}(x)$ of $x_{1}+\cdots+x_{n}$ is identical with $\bar{W}_{n}(u)$ if the relation

$$
u=\left(x-A_{n}\right) / B_{n}^{1 / 2}
$$

between $u$ and $x$ is valid.

According to (19) and $W_{n}(x)=\bar{W}_{n}(u)$ we have uniformly in $x$

$$
W_{n}(x)=\phi\left(\frac{x-A_{n}}{\left(2 B_{n}\right)^{1 / 2}}\right)+\sigma, \quad|\sigma|<\epsilon .
$$

We can thus say: To every positive $\epsilon$ there is a positive $\delta$, so that (21) holds true, if the assumptions $\left(4^{\prime}\right)$ are satisfied. 
We call special attention to the case

$$
0<g<b_{i} ; \quad c_{i}<H,
$$

$g$ and $H$ being independent of $i$. One has $g n<B_{n}$ and as for the rest $b_{i}<c_{i}^{2 / 3}<H^{2 / 3}$ and $B_{n}<n H^{2 / 3}$, so that $B_{n}$ is exactly of the order $n$. It follows that

$$
\frac{c_{i}}{b_{i} B_{n}^{1 / 2}}<\frac{H}{g g^{1 / 2} n^{1 / 2}} .
$$

Therefore $\left(4^{\prime}\right)$ can be satisfied, if $n$ is sufficiently large.

In the same way one can discuss sets of chance variables $x_{11}, x_{12}, \cdots, x_{1 n_{1}}, \cdots, x_{k 1}, x_{k 2}, \cdots, x_{k n_{k}}, \cdots, n_{k}$ increasing indefinitely with $k$. It is supposed that the quotients

$$
\frac{c_{k 1}}{b_{k 1} B_{n_{k}}^{1 / 2}}, \ldots, \frac{c_{k n_{k}}}{b_{k n_{k}} B_{n_{k}}^{1 / 2}}
$$

approach 0 as a limit, if $k$ increases indefinitely. That means that if any $\delta>0$ is given one can choose $k$ sufficiently large so that all these quotients are less than $\delta$. Then the distribution function $W_{n_{k}}$ of $x_{k 1}+\cdots+x_{k n_{k}}$ approaches $\phi\left(\left(x-A_{n_{k}}\right) /\left(2 B_{n_{k}}\right)^{1 / 2}\right)$ as a limit.

The conditions for the quotients are in particular satisfied if $B_{n_{k}}>B>0$ for every $k$ ( $B$ being a constant number) and the quotients $c_{k 1} / b_{k 1}, \cdots, c_{k n_{k}} / b_{k n_{k}}$ approach 0 , as $k$ increases indefinitely.

NEW YORK, N. Y. 